\title{
Photometric and period analysis of the eclipsing binary system V2783 Ori
}

\author{
İ. Bulut ${ }^{1,3}$ and A. Bulut ${ }^{2,3}$ \\ ${ }^{1}$ Department of Space Sciences and Technologies, Faculty of Arts and \\ Sciences, Çanakkale Onsekiz Mart University, Terzioğlu Kampüsü, \\ TR-17020, Canakkale, Turkey TR-17020, Çanakkale, Turkey; \\ (E-mail: ibulut@comu.edu.tr) \\ 2 Department of Physics, Faculty of Arts and Sciences, Çanakkale Onsekiz \\ Mart University, Terzioğlu Kampüsü, TR-17020, Çanakkale, Turkey \\ 3 Astrophysics Research Centre and Observatory, Chanakkale Onsekiz Mart \\ University, Terzioğlu Kampüsü, TR-17020, Çanakkale, Turkey
}

Received: March 5, 2021; Accepted: May 7, 2021

\begin{abstract}
In this study we performed the first detailed photometric and period analysis of the recently discovered eclipsing binary star V2783 Ori. The analysis of the light curve has shown that the system is in a detached configuration, with the orbital eccentricity $e=0.274 \pm 0.008$. The photometric mass ratio of V2783 Ori determined from the light curve analysis is $q=0.734 \pm 0.015$. The absolute parameters of the system were obtained as a result of the light curve solution. Absolute parameters were found as follows: masses $M_{1}=1.80 \pm 0.01$ $\mathrm{M}_{\odot}, M_{2}=1.32 \pm 0.01 \mathrm{M}_{\odot}$, radii $R_{1}=2.19 \pm 0.10 \mathrm{R}_{\odot}, R_{2}=1.91 \pm 0.10$ $\mathrm{R}_{\odot}$, temperatures $T_{1}=8325 \pm 100 \mathrm{~K}$ and $T_{2}=8300 \pm 100 \mathrm{~K}$. A comparison of these parameters with theoretical stellar models show that the secondary component is located inside the main-sequence band and still close to ZAMS, while the primary component is located in the middle of ZAMS-TAMS limits. The distance of the system determined from the absolute parameters, $d=1064$ $\pm 150 \mathrm{pc}$, takes into account the interstellar reddening.

The $(\mathrm{O}-\mathrm{C})$ diagram was discussed considering all reliable minima times found in the literature and new values for the parameters of the apsidal motion and light-time effect were found. The apsidal motion rate of V2783 Ori is $\dot{\omega}=$ $0.0063 \pm 0.0017 \mathrm{deg}$ cycle $^{-1}$, and it corresponds to an apsidal motion period of $U=656 \pm 103 \mathrm{yr}$. The third body orbital period is $10 \pm 2 \mathrm{yr}$, its minimal mass is $0.82 \pm 0.11 \mathrm{M}_{\odot}$.
\end{abstract}

Key words: photometric — stars: variables: binaries: eclipsing — stars: individual: V2783 Ori

\section{Introduction}

It is well known that in close binary systems of stars with eccentric orbits, the tidal and rotational distortions of the components from spherical symmetry lead 
to secular changes in the position of the periastron.

Apsidal motion is the rotation of the major orbital axis of an eccentric orbital binary star in space. It is primarily caused by the effect of the tidal bulge due to the companion and the rotational distortions of the stars. Besides these contributions to the apsidal motion, there is also a general relativistic contribution. This contribution is often small.

Apsidal motion studies on the eclipsing binaries allow the determination of the observational internal constants of stars and the observational tests of General Relativity theory (Giménez 1985; Claret \& Giménez 1993). The orbital elements of the binary stars with eccentric orbits have been used to constrain the timescales of synchronization and circularization predicted by the tidal theories (Zahn 2008). These studies depend on the number of such systems and the precision of their absolute and orbital parameters.

The star system V2783 Ori (GSC 0143-0226, HD 252984, $P=4.21618, V$ $\left.=10^{m} .39\right)$ is identified as a new eclipsing binary by Otero et al. (2004). They determined its period as 4.21618 days. The spectral type of this system was classified as A0 (according to Otero et al. (2004)). The ASAS light curve of V2783 Ori indicates that the orbit is eccentric, with the secondary minimum occurring at phase 0.54 . The apsidal motion in the system was calculated by Bulut et al. (2017) who obtained its period to be $U=415 \mathrm{yr}$ and the orbital eccentricity $e=0.25$. The Gaia EDR3 gives a parallax of $0.876 \pm 0.035$ mas for the system, which means a distance of $1141 \pm 46 \mathrm{pc}$ (Gaia Collaboration et al. 2021).

There has been no photometric and spectroscopic study for V2783 Ori until now. In this paper, the detailed photometric and the orbital period study of V2783 Ori is presented.

\section{Observational data}

V2783 Ori was named as ASAS 061057+0621.1 in the ASAS catalogue of variable stars. We have taken from the ASAS-3 database (Pojmanski 2003) the $V$ photometric data of V2783 Ori observed between 2002, August, and 2009, November. About 476 observations of the system were made during the survey, of which 442 were recorded as being of good photometric quality; these data were used in the analysis. The light curve of the system is plotted in Figure 1. The phases are computed according to the following linear light elements given by Otero et al. (2004):

$$
H J D(\text { Min } I)=2412946.750+4^{\mathrm{d}} .21618 \times E .
$$




\section{Analysis of the light curves}

For the analysis of the light curve of V2783 Ori we used the program PHOEBE (version 0.31a), which was developed by Prša \& Zwitter (2005). This software is based on the Wilson-Devinney (W-D) method (Wilson \& Devinney 1971). The program computes the light curves as a function of the following main parameters: orbital eccentricity $(e)$, longitude of periastron $(\omega)$, orbital inclination $(i)$, surface temperature $\left(T_{1,2}\right)$, dimensionless surface potentials $\left(\Omega_{1,2}\right)$, mass ratio $\left(q=M_{2} / M_{1}\right)$, relative luminosities $\left(L_{1,2}\right)$, gravity darkening exponents $\left(g_{1,2}\right)$, limb darkening coefficients $\left(x_{1,2}\right)$ and bolometric albedos $\left(A_{1,2}\right)$.

The detached binary mode (in W-D mode 2) was used with several assumptions. Some parameters of components should be fixed during the light-curve modeling. The corresponding linear limb-darkening coefficients were interpolated for the square root law from van Hamme's tables (van Hamme 1993). The values of gravity-darkening exponents $\left(g_{1,2}=1.0\right)$ and bolometric albedo coefficients $\left(A_{1,2}=1.0\right)$ were set at their suggested values for the radiative atmospheres (Lucy 1968).

The magnitudes and color indices in the literature can be used to estimate the mean effective temperature of the system for the combined light of the system. The results were collected in Table 1 . We used the color/temperature calibrations of Casagrande et al. (2010) and Ramírez \& Meléndez (2005) for the dwarf stars. The weighted average of the seven estimates is $8325 \mathrm{~K}$. This value is adopted to be the mean of the effective temperature of the primary star in the analyses.

Table 1. Photometric indices and inferred mean effective temperature of V2783 Ori.

\begin{tabular}{lccc}
\hline \hline Photometric Index & Value & $T(\mathrm{~K})$ & References \\
\hline Johnson $V$ & $10.39 \pm 0.05$ & & 1 \\
2MASS $J$ & $10.003 \pm 0.024$ & & 2 \\
2MASS $H$ & $10.008 \pm 0.026$ & & 2 \\
2MASS $K s$ & $9.985 \pm 0.016$ & & 2 \\
Johnson $B-V$ & $-0.04 \pm 0.05$ & $9210 \pm 800$ & 1 \\
Johnson/2MASS $V-J$ & $0.387 \pm 0.036$ & $8254 \pm 768$ & 1,2 \\
Johnson/2MASS $V-H$ & $0.382 \pm 0.039$ & $8117 \pm 829$ & 1,2 \\
Johnson/2MASS $V-K s$ & $0.387 \pm 0.033$ & $8343 \pm 711$ & 1,2 \\
2MASS $J$ - $K s$ & $0.018 \pm 0.019$ & $7750 \pm 818$ & 2 \\
\hline \hline
\end{tabular}

1: Høg et al. (2000), 2: Cutri et al. (2003)

As the spectroscopic value of the mass ratio is not known, we applied the $q$-search method with a step of 0.1 with trials, to find sensible photometric 
estimates for the mass ratio. Figure 2 presents the sum of the squared residuals $\sum(O-C)^{2}$ for the tested mass ratios $(q)$. It can be seen that the smallest value of squared residuals is achieved around $q=0.75$. This value was used as the initial value of the mass radio in differential-corrections.

Considering the calculated apsidal motion period given in Table 3 , the total change in the longitude of periastron during the observation time interval can be neglected. Therefore we assumed $\dot{\omega}=0.0$ in the analyses.

The adjustable parameters are the temperature of secondary star $\left(T_{2}\right)$, the orbital inclination $(i)$, the orbital eccentricity $(e)$, the longitude of periastron $(\omega)$, the surface potentials $\left(\Omega_{1,2}\right)$, the relative luminosity of primary star $\left(L_{1}\right)$, and the mass ratio $(q)$. The results of the light curve solution can be found in Table 2. For the parameters in the light curve fitting, the standard deviations of the differential corrections supplied by the W-D program were used as an error. The synthetic light curve and the observations are shown in Figure 1.

The analysis of the light curve suggests partial eclipses in both of the light curve minima. The depths of the primary and secondary minima are 0.581 mag and $0.523 \mathrm{mag}$ in $V$, respectively. The duration of the minima are also, respectively, 4.84 hours for the primary and 9.29 hours for the secondary.

Table 2. Parameters of the light curves of V2783 Ori.

\begin{tabular}{lc}
\hline \hline Parameter & ASAS $(V)$ \\
\hline$T$ (HJD) & $24412949.828 \pm 0.013$ \\
$P$ (day) & $4.216180 \pm 0.000001$ \\
$i$ (deg) & $87.18 \pm 0.09$ \\
$e$ & $0.274 \pm 0.008$ \\
$\omega($ deg $)$ & $70.28 \pm 0.70$ \\
PSHIFT & $0.0213 \pm 0.0004$ \\
$T_{1}(\mathrm{~K})$ & 8325 \\
$T_{2}(\mathrm{~K})$ & $8300 \pm 57$ \\
$\Omega_{1}$ & $8.40 \pm 0.11$ \\
$\Omega_{2}$ & $8.22 \pm 0.15$ \\
$q$ & $0.734 \pm 0.015$ \\
$x_{1}$ & 0.503 \\
$x_{2}$ & 0.503 \\
$A_{1}=A_{2}$ & 1.0 \\
$g_{1}=g_{2}$ & 1.0 \\
$L_{1}\left(L_{1} / L_{\text {Total }}\right)$ & $0.59 \pm 0.11$ \\
$L_{2}\left(L_{2} / L_{\text {Total }}\right)$ & 0.41 \\
$r_{\text {mean }, 1}$ & $0.136 \pm 0.002$ \\
$r_{\text {mean }, 2}$ & $0.112 \pm 0.002$ \\
\hline \hline
\end{tabular}




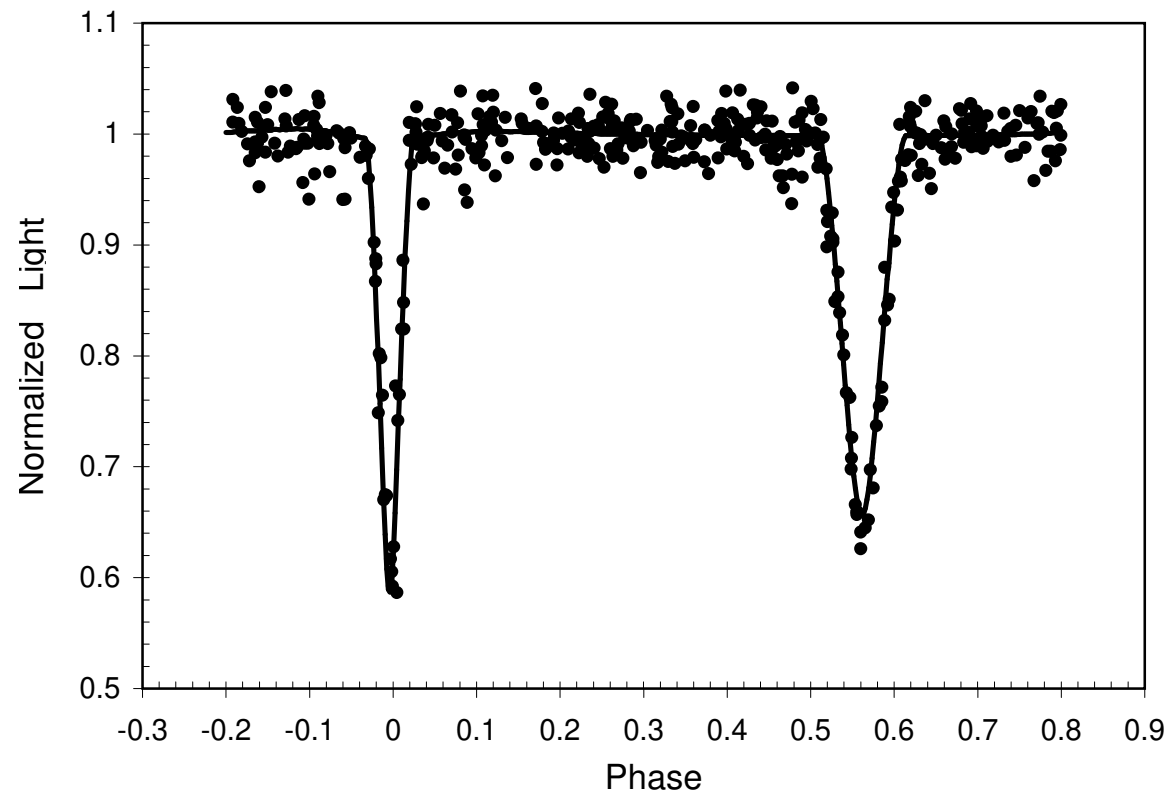

Figure 1. Light curves of V2783 Ori. The points are the individual observations. The solid lines are the synthetic light curves based on the parameters in Table 2 .

\section{4. $(\mathrm{O}-\mathrm{C})$ analysis}

The orbital period variation of V2783 Ori was studied by means of an (O-C) diagram analysis. The minima times were taken from the modern database of the (O-C) Gateway (http://var2.astro.cz/ocgate/) (Paschke \& Brat 2006). A total number of 25 photoelectric and CCD times of minimum light were collected, including 15 primary and 10 secondary eclipses. Those compiled minimum times spread over 18 years from 1999 to 2017. These minima times were used to study $(\mathrm{O}-\mathrm{C})$ residuals of $\mathrm{V} 2783$ Ori. The $(\mathrm{O}-\mathrm{C})$ values were computed with the linear ephemeris which was given in Eq.1.

The corresponding $(\mathrm{O}-\mathrm{C})$ curve is shown in Figure 3. It is clearly evident that the (O-C) diagram of V2783 Ori is represented by a sinusoidal variation superimposed on the apsidal motion effect. We assumed that the most likely cause of the sinusoidal variation could be an unseen third body in the system.

The calculations of the apsidal motion rate of the system were made with the computer program written by Zasche et al. (2009), which uses the method described by Giménez \& Garcia-Pelayo (1983), with equations revised by Giménez 


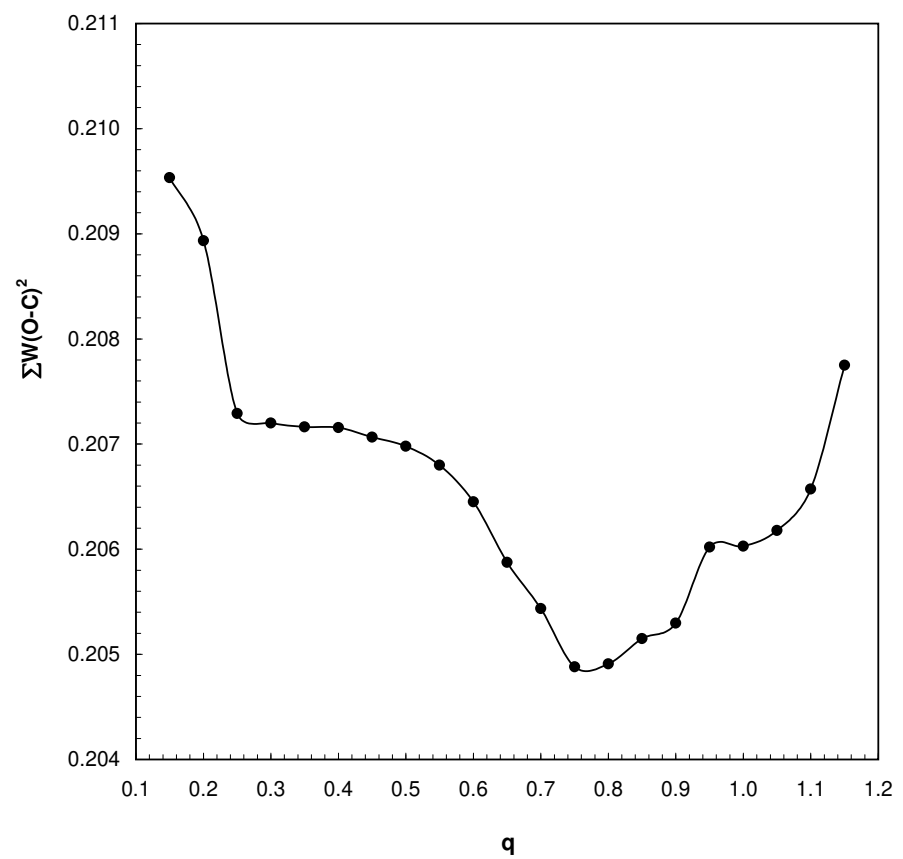

Figure 2. Sum of the squared residuals as a function of the mass ratio for V2783 Ori.

\& Bastero (1995). The standard approach formulated by Irwin (1959) was used for the light-time effect resulting from the effect of the third body in the system.

Parameters are determined in the following procedures :

For the apsidal motion;

$T_{\circ}$ : The zero epoch (HJD);

$\omega_{\mathrm{o}}$ : The position of the periastron at the zero epoch;

$P_{a}:$ The anomalistic period;

$P_{s}$ : The sidereal period;

e: The orbital eccentricity;

$\dot{\omega}$ : The apsidal motion rate;

$\mathrm{U}$ : The apsidal motion period $\left(\mathrm{U}=360 P_{a} / \dot{\omega}\right)$;

For the light-time effect

A: The observed semi-amplitude of the light-time curve;

$T_{3}$ : The time of the periastron passage of the third body;

$a_{12} \sin i_{3}$ : The semi-major axis of the relative orbit of the eclipsing pair around the common center of mass;

$e_{3}$ : The eccentricity of the third-body orbit;

$\omega_{3}$ : The longitude of periastron of the third-body orbit.

The computed apsidal motion and the third body parameters with their 
errors are presented in Table 3. The observational curves and the theoretical best fit curves, as well as the residuals, are plotted in Figures 3-4.

The apsidal motion rate $(\dot{\omega})$ obtained in this study appears to be statistically significant, $0.0063 \pm 0.0017 \mathrm{deg}$ cycle $^{-1}$. This corresponds to an apsidal period of $U=656 \pm 103 \mathrm{yr}$. Using the parameters of the hypothetical three-body orbit in Table 3, we derived that the mass of the third body in the system is $0.82 \pm$ $0.11 \mathrm{M}_{\odot}$ for $i_{3}=90 \mathrm{deg}$.

Table 3. Apsidal motion and third body parameters for V2783 Ori.

\begin{tabular}{lll}
\hline \hline Parameter & Unit & Value \\
\hline$T_{0}$ & (HJD) & $2452946.879 \pm 0.046$ \\
$P_{s}$ & (day) & $4.21617 \pm 0.00007$ \\
$P_{a}$ & (day) & $4.21625 \pm 0.00007$ \\
$e$ & & $0.253 \pm 0.016$ \\
$\dot{\omega}$ & $(\mathrm{deg} /$ cycle $)$ & $0.0063 \pm 0.0017$ \\
$\omega_{0}$ & $(\mathrm{deg})$ & $64.3 \pm 3.2$ \\
$U$ & $(\mathrm{yr})$ & $656 \pm 103$ \\
\hline$A$ & $(\mathrm{day})$ & $0.0092 \pm 0.0046$ \\
$T_{3}$ & $(\mathrm{HJD})$ & $2454100 \pm 2100$ \\
$P_{3}$ & $(\mathrm{yr})$ & $10 \pm 2$ \\
$e_{3}$ & & $0.262 \pm 0.087$ \\
$\omega_{3}$ & $(\mathrm{deg})$ & $65.7 \pm 2.3$ \\
\hline$a_{12} \sin i_{3}$ & $(\mathrm{au})$ & $1.6 \pm 0.8$ \\
$f\left(M_{3}\right)$ & $\left(\mathrm{M}_{\odot}\right)$ & $0.043 \pm 0.025$ \\
$M_{3 \min }$ & $\left(\mathrm{M}_{\odot}\right)$ & $0.82 \pm 0.11$ \\
\hline \hline
\end{tabular}

\section{Conclusions and remarks}

The light curve of the eclipsing binary V2783 Ori has been modeled using the W-D method. The analysis has shown that V2783 Ori is a detached binary system with a moderately eccentric orbit.

The absolute parameters of the system cannot be determined directly because no spectroscopic observations were available. But the absolute dimensions for V2783 Ori can be estimated from the empirical relations between temperature and stellar mass. According to the temperature of the primary component, its main sequence mass would be $M_{1}=1.80 \pm 0.01 M_{\odot}$ (see Eker et al. (2015)). In this case the mass of the secondary component should be $M_{2}=1.32 \pm 0.01$ $M_{\odot}$ using the mass ratio $(q=0.734 \pm 0.015)$ given in Table 2 . The distance between the mass centers of the components would be calculated as $a=16.1$ 


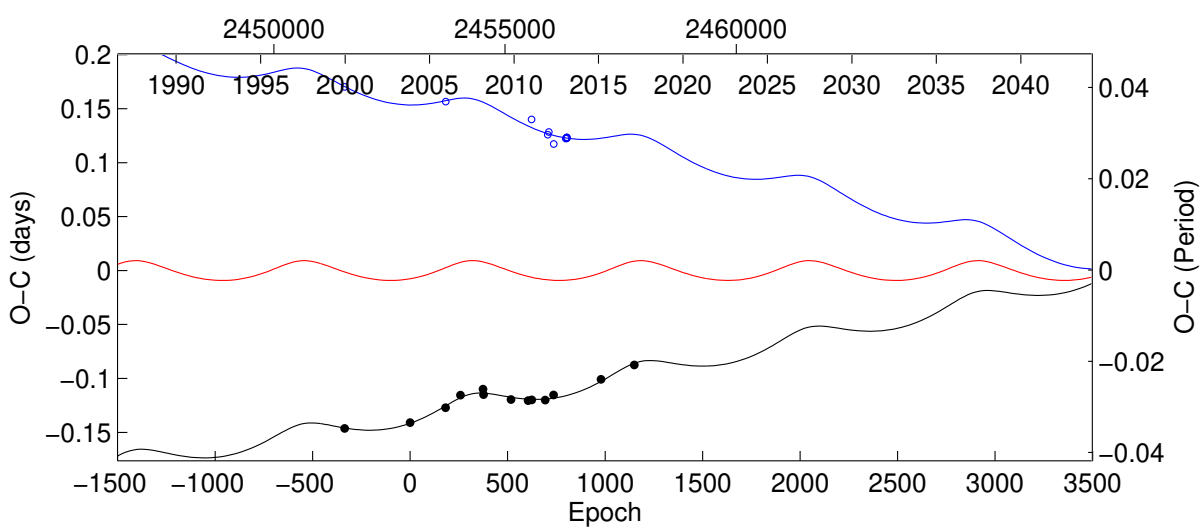

Figure 3. The complete $(\mathrm{O}-\mathrm{C})$ diagram for mid-eclipse times of V2783 Ori. The solid lines denote combination of the apsidal motion and the third-body LITE. Filled and open symbols represent the individual primary and secondary minima, respectively.

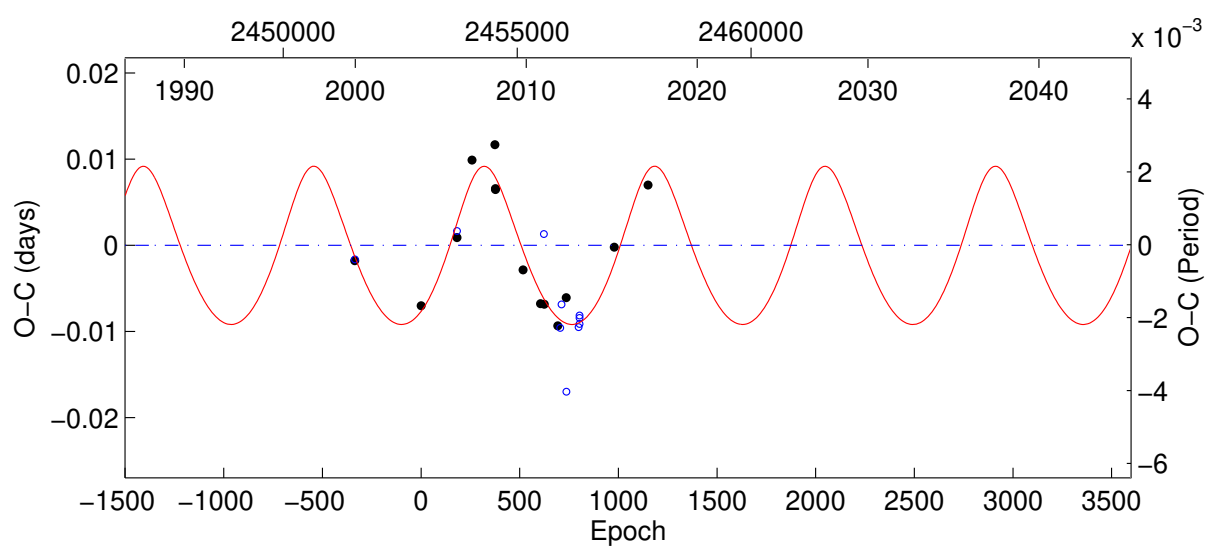

Figure 4. The (O-C) diagram of V2783 Ori after subtraction of apsidal motion.

$\pm 0.2 R_{\odot}$ using Kepler's Third Law and the orbital period $\left(P=4^{d} .21617\right)$. By using definition of fractional radii as $r_{1,2}=R_{1,2} / a$ and the mean fractional radii from Table 2 as $r_{1}=0.136 \pm 0.002$ and $r_{2}=0.112 \pm 0.002$, the radius of the primary and secondary components $R_{1}=2.19 \pm 0.10 R_{\odot}, R_{2}=1.91 \pm 0.10 R_{\odot}$ can be obtained. The absolute dimensions are listed in Table 3 . The bolometric corrections (BC) have been adopted from Flower (1996), together with $\mathrm{M}_{\odot, \text { bol }}$ $=4.75$, according to their effective temperatures. 
Table 4. The absolute parameters of V2783 Ori.

\begin{tabular}{lcc}
\hline \hline Parameter & Primary & Secondary \\
\hline$M\left(\mathrm{M}_{\odot}\right)$ & $1.80 \pm 0.01$ & $1.32 \pm 0.01$ \\
$R\left(\mathrm{R}_{\odot}\right)$ & $2.19 \pm 0.10$ & $1.91 \pm 0.10$ \\
$\log g(\mathrm{cgs})$ & $4.01 \pm 0.10$ & $4.00 \pm 0.10$ \\
$T(\mathrm{~K})$ & $8325 \pm 100$ & $8300 \pm 100$ \\
$\log L\left(\mathrm{~L}_{\odot}\right)$ & $1.61 \pm 0.02$ & $1.55 \pm 0.02$ \\
$M_{b o l}$ & $0.80 \pm 0.05$ & $1.10 \pm 0.06$ \\
$a\left(\mathrm{R}_{\odot}\right)$ & $16.1 \pm 0.2$ & \\
B.C. & -0.17 & -0.16 \\
$M_{v}$ & $0.97 \pm 0.04$ & $1.26 \pm 0.04$ \\
$d(\mathrm{pc})$ & $1064 \pm 150$ & \\
\hline \hline
\end{tabular}

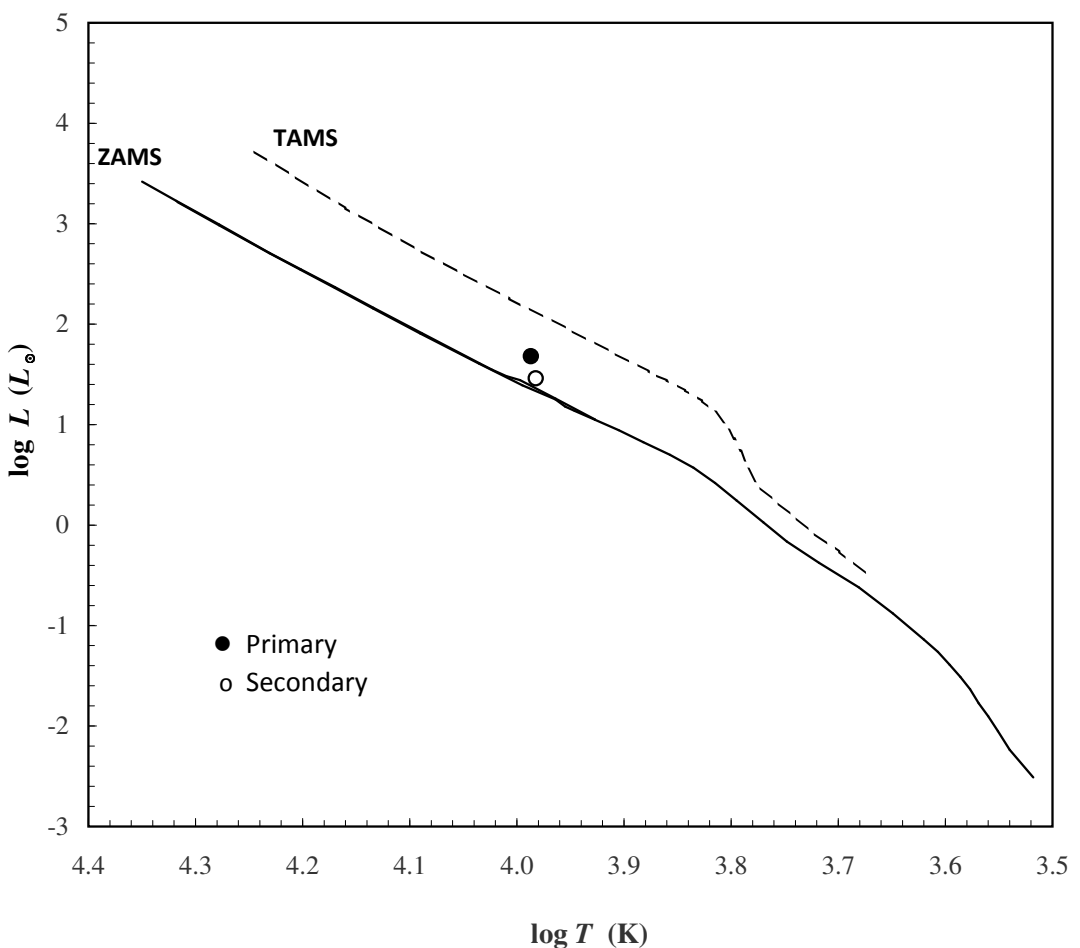

Figure 5. Locations of the components of V2783 Ori in the HR diagram. The ZAMS (lower) and the TAMS (upper) lines were taken from (Girardi et al. 2000) for the solar chemical composition. The filled and open symbols represent the primary and secondary stars, respectively. 
Computed absolute elements of both the stars, reported in Table 4, are also used to estimate the evolutionary status of V2783 Ori by means of the $\log T-\log$ $L$ (i.e. Hertzsprung-Russell) diagram (in Fig. 5). The Zero Age Main Sequence (ZAMS) and the Terminal Age Main Sequence (TAMS) were obtained from Girardi et al. (2000). As it can be seen from this figure, the primary component of the system is located almost in the middle of ZAMS-TAMS limits, while the secondary component is found to be closer to the ZAMS. According to the mass values of these stars, it seems that the more massive component is more evolved that the less massive one.

The estimate of the interstellar reddening was performed by using maps of dust infrared emission by Schlafly \& Finkbeiner (2011). We obtained a colour excess of $E_{(B-V)}=0.316 \pm 0.006$. Using the colour excess and the apparent visual magnitude of $V=10.39$ at maximum light, we computed the distance to the V2783 Ori system to be $1064 \pm 150 \mathrm{pc}$. This result agrees with the value of $1141 \pm 46 \mathrm{pc}$ obtained by the trigonometric parallax $(0.876 \pm 0.035$ mas $)$ from Gaia EDR3 (Gaia Collaboration et al. 2021).

Analysing the (O-C) diagram, we solved the apsidal motion and the lighttime effect (LITE) simultaneously. The apsidal motion rate $(\dot{\omega})$ obtained in this study appears to be statistically significant, $0.0063 \pm 0.0017 \mathrm{deg}$ cycle $^{-1}$. This corresponds to an apsidal period of $U=656 \pm 103 \mathrm{yr}$. The observational average value $\left(\log k_{2, o b s}\right)$ of the internal structure constant can be calculated from the apsidal motion period with the absolute dimensions. We found the general relativistic contribution within the total apsidal motion rate, approximately $7.6 \%$ of the observed value. After making this correction, we calculated $\log k_{2, o b s}=$ 2.04 with an assumption of the periastron-synchronization.

Using the standard solar composition $(\mathrm{Y}, \mathrm{Z})=(0.28,0.02)$ models of Claret (2004), we obtained an average theoretical internal structure constant value $k_{2, t e o}=2.40$ by the interpolation. According to these results, the observational mean central density concentration for V2783 Ori seems to be smaller than that predicted by Claret (2004) models.

The invisible component around the V2783 Ori has a period of $P_{3}=10 \pm$ $2 \mathrm{yr}$, an eccentricity of $e_{3}=0.262 \pm 0.087$, and its mass is $M_{3}=0.82 \pm 0.19$ $\mathrm{M}_{\odot}$, which could be a main-sequence star. The third-body mass corresponds to a spectral type of about $\mathrm{K} 5.5 \mathrm{~V}$, and the bolometric luminosity is calculated to be $L_{3}=0.36 \mathrm{~L}_{\odot}$ (see Eker et al. (2015); Pecaut \& Mamajek (2013)), which contributes about $0.6 \%$ to the total luminosity of V2783 Ori. This contribution is negligibly small.

Future spectroscopic observations will help to a more complete understanding of the system.

Acknowledgements. The author would like to thank an anonymous referee who provided valuable comments for improving the manuscript. 


\section{References}

Bulut, A., Bulut, I., Ciçek, C., \& Erdem, A., Apsidal motion of two eclipsing binaries: V796 Cyg and V2783 Ori. 2017, in American Institute of Physics Conference Series, Vol. 1815, Turkish Physical Society 32nd International Physics Congress, 080007

Casagrande, L., Ramírez, I., Meléndez, J., Bessell, M., \& Asplund, M., An absolutely calibrated $\mathrm{T}_{\text {eff }}$ scale from the infrared flux method. Dwarfs and subgiants. 2010, Astronomy and Astrophysics, 512, A54, DOI: 10.1051/0004-6361/200913204

Claret, A., New grids of stellar models including tidal-evolution constants up to carbon burning. I. From 0.8 to $125 \mathrm{M}_{\odot}$ at $\mathrm{Z}=0.02$. 2004, Astronomy and Astrophysics, 424, 919, DOI: 10.1051/0004-6361:20040470

Claret, A. \& Giménez, A., The apsidal motion test of the internal stellar structure: comparison between theory and observations. 1993, Astronomy and Astrophysics, 277, 487

Cutri, R. M., Skrutskie, M. F., van Dyk, S., et al., VizieR Online Data Catalog: 2MASS All-Sky Catalog of Point Sources (Cutri+ 2003). 2003, VizieR Online Data Catalog, II $/ 246$

Eker, Z., Soydugan, F., Soydugan, E., et al., Main-Sequence Effective Temperatures from a Revised Mass-Luminosity Relation Based on Accurate Properties. 2015, Astronomical Journal, 149, 131, DOI: 10.1088/0004-6256/149/4/131

Flower, P. J., Transformations from Theoretical Hertzsprung-Russell Diagrams to Color-Magnitude Diagrams: Effective Temperatures, B-V Colors, and Bolometric Corrections. 1996, Astrophysical Journal, 469, 355, DOI: 10.1086/177785

Gaia Collaboration, Brown, A. G. A., Vallenari, A., et al., Gaia Early Data Release 3. Summary of the contents and survey properties. 2021, Astronomy and Astrophysics, 649, A1, DOI: 10.1051/0004-6361/202039657

Giménez, A., General-relativistic periastron advances in eclipsing binary systems. 1985, Astrophysical Journal, 297, 405, DOI: 10.1086/163539

Giménez, A. \& Bastero, M., A Revision of the Ephemeris-Curve Equations for Eclipsing Binaries with Apsidal Motion. 1995, Astrophysics and Space Science, 226, 99, DOI: $10.1007 / \mathrm{BF} 00626903$

Giménez, A. \& Garcia-Pelayo, J. M., A New Method for the Analysis of Apsidal Motions in Eclipsing Binaries. 1983, Astrophysics and Space Science, 92, 203, DOI: 10.1007/BF00653602

Girardi, L., Bressan, A., Bertelli, G., \& Chiosi, C., Evolutionary tracks and isochrones for low- and intermediate-mass stars: From 0.15 to $7 \mathrm{M}_{\text {sun }}$, and from $\mathrm{Z}=0.0004$ to 0.03. 2000, Astronomy and Astrophysics, Supplement, 141, 371, DOI: 10.1051/aas:2000126

$\mathrm{H} \varnothing \mathrm{g}$, E., Fabricius, C., Makarov, V. V., et al., The Tycho-2 catalogue of the 2.5 million brightest stars. 2000, Astronomy and Astrophysics, 355, L27

Irwin, J. B., Standard light-time curves. 1959, Astronomical Journal, 64, 149, DOI: $10.1086 / 107913$ 
Lucy, L. B., The Structure of Contact Binaries. 1968, Astrophysical Journal, 151, 1123, DOI: $10.1086 / 149510$

Otero, S. A., Wils, P., \& Dubovsky, P. A., New Eclipsing Binaries Found in the NSVS Database I. 2004, Information Bulletin on Variable Stars, 5570, 1

Paschke, A. \& Brat, L., O-C Gateway, a Collection of Minima Timings. 2006, Open European Journal on Variable Stars, 23, 13

Pecaut, M. J. \& Mamajek, E. E., Intrinsic Colors, Temperatures, and Bolometric Corrections of Pre-main-sequence Stars. 2013, Astrophysical Journal, Supplement, 208, 9, DOI: 10.1088/0067-0049/208/1/9

Pojmanski, G., The All Sky Automated Survey. The Catalog of Variable Stars. II. $6^{\mathrm{h}}-12^{\mathrm{h}}$ Quarter of the Southern Hemisphere. 2003, Acta Astronomica, 53, 341

Prša, A. \& Zwitter, T., A Computational Guide to Physics of Eclipsing Binaries. I. Demonstrations and Perspectives. 2005, Astrophysical Journal, 628, 426, DOI: $10.1086 / 430591$

Ramírez, I. \& Meléndez, J., The Effective Temperature Scale of FGK Stars. II. $\mathrm{T}_{\text {eff }}$ :Color: $[\mathrm{Fe} / \mathrm{H}]$ Calibrations. 2005, Astrophysical Journal, 626, 465, DOI: $10.1086 / 430102$

Schlafly, E. F. \& Finkbeiner, D. P., Measuring Reddening with Sloan Digital Sky Survey Stellar Spectra and Recalibrating SFD. 2011, Astrophysical Journal, 737, 103, DOI: $10.1088 / 0004-637 \mathrm{X} / 737 / 2 / 103$

van Hamme, W., New Limb-Darkening Coefficients for Modeling Binary Star Light Curves. 1993, Astronomical Journal, 106, 2096, DOI: 10.1086/116788

Wilson, R. E. \& Devinney, E. J., Realization of Accurate Close-Binary Light Curves: Application to MR Cygni. 1971, Astrophysical Journal, 166, 605, DOI: 10.1086/150986

Zahn, J. P., Tidal dissipation in binary systems. 2008, in EAS Publications Series, Vol. 29, EAS Publications Series, ed. M. J. Goupil \& J. P. Zahn, 67-90

Zasche, P., Liakos, A., Niarchos, P., et al., Period changes in six contact binaries: WZ And, V803 Aql, DF Hya, PY Lyr, FZ Ori, and AH Tau. 2009, New Astronomy, 14, 121, DOI: 10.1016/j.newast.2008.06.002 